\title{
Two-dimensional time resolved measurements of toroidal velocity correlated with density blobs in magnetized plasmas
}

\author{
B. Labit, ${ }^{1}$ I. Furno, ${ }^{1}$ M. Podestà, ${ }^{2}$ and A. Fasoli ${ }^{1}$ \\ ${ }^{1}$ Centre de Recherches en Physique des Plasmas (CRPP), Ecole Polytechnique Fédérale de Lausanne (EPFL), \\ Association Euratom-Confédération Helvétique, CH-1015 Lausanne, Switzerland \\ ${ }^{2}$ Department of Physics and Astronomy, University of California, Irvine, California 92697, USA
}

(Received 13 May 2008; accepted 7 July 2008; published online 4 August 2008)

\begin{abstract}
A new method for toroidal velocity measurements with Mach probes is presented. This technique is based on the conditional sampling technique, the triggering events being density blobs. A reconstruction of the time resolved two-dimensional profile of electron density, electron temperature, plasma potential, and toroidal velocity is possible with a single point measurement on a shot-to-shot basis. (C) 2008 American Institute of Physics. [DOI: 10.1063/1.2965141]
\end{abstract}

Flows are ubiquitous in magnetic fusion plasmas. Their origin and their possible role on the confinement quality are still open questions. In the edge plasma, only measurements of the Mach numbers at few points are usually performed using directional Mach probes. ${ }^{1,2}$ This diagnostic consists of two electrostatic probes separated by an insulator. The two tips measure the currents collected parallel and antiparallel to the magnetic field.

The goal of this Note is to present a novel technique, based on the conditional sampling technique, ${ }^{3}$ to extract time resolved Mach number and time resolved electron temperature from simultaneous measurements at the same location. The experiments reported herein are performed on TORPEX (Ref. 4) (major radius $R=1 \mathrm{~m}$, minor radius $a=0.2 \mathrm{~m}$ ), a simple toroidal plasma, confined by a primarily toroidal magnetic field, with no induced current or rotational transform. In such configuration no trapped particle or magnetic shear effects are present and, due to the low plasma pressures, electromagnetic and $\beta$ effects need not be taken into account (typically, $\beta=2 \mu_{0} n T / B^{2} \leqslant 10^{-4}$ ). Hydrogen plasmas are produced by means of microwaves in the electron cyclotron (EC) range of frequencies. ${ }^{5}$ A low level of EC power $P_{\mathrm{EC}} \simeq 400 \mathrm{~W}$ is used. A vertical magnetic field $\left|B_{z}\right|$ $=2.3 \mathrm{mT}$ is superposed on a toroidal magnetic field of $\left|B_{\phi}\right|$ $=80.6 \mathrm{mT}$ on axis, thus resulting in helicoidal magnetic field lines with both ends terminating on the torus vessel. This experimental setup gives rise to a plasma source profile localized around $r=-3 \mathrm{~cm}$ (the EC resonance is located at $r=-12 \mathrm{~cm}$ ), which ensures negligible plasma production for $r>6 \mathrm{~cm}$ and results in vertically elongated plasma profiles.

In order to measure flows, a Mach probe has been built and installed on TORPEX. It consists of a Teflon cube $(1 \mathrm{~cm}$ each side). On five of the six faces, a tungsten plate (diameter: $8 \mathrm{~mm}$ ) is glued. Each plate can be operated as a standard Langmuir probe (sweep mode, biased or floating). A ceramic tube, glued on the sixth face at one end and screwed on a stainless steel tube at the other end, supports the probe inside the plasma. A schematic drawing of the Mach probe is represented in Fig. 1(a). According to this geometry, the toroidal mach number is deduced from measurements of the upstream and downstream currents on faces 1 and 5, respectively. The Mach probe is mounted on a two-dimensional
(2D) poloidally moving system allowing measurements of the toroidal Mach number over a large fraction of the cross section [Fig. 1(b)]. ${ }^{6}$

Each tip of the probe is connected to a dedicated electronic module from which the raw signals are sent to the acquisition. The acquisition systems are connected to an Ethernet link, allowing us to download the data into the MDSplus database. Each module represents a node of a bus, based on the BitBus protocol, which manages the flow of information from and to the different TORPEX subsystems. Through the BitBus it is possible to set remotely the bias voltage applied to the probes and the sensitivity of the current measurement. Mach probe signals are acquired on channels of a 96-channel D-TACQ digitizer, running at $250 \mathrm{ksamples} / \mathrm{s}$. This frequency has been chosen on the basis of the typical time scales of the phenomena observed in TORPEX plasmas, which are usually below $10 \mathrm{kHz}$.

An abundant literature concerning the theory of flow measurements with Mach probes exists (a good introduction is Stangeby's textbook ${ }^{7}$ ). Taking a commonly accepted definition of the Mach number given by Refs. 8 and 9, the time averaged fluid velocity can be defined as

$$
V \equiv C_{s} M=C_{s} K \log \left(\frac{\bar{I}_{\text {up }}}{\bar{I}_{\text {down }}}\right),
$$

where $C_{s} \equiv \sqrt{Z k_{B} T_{e} / m_{i}}$ is the ion sound speed ( $Z$ is the charge number, $k_{B}$ is the Boltzmann constant, and $m_{i}$ is the ion mass), and $\bar{I}_{\text {up }}$ and $\bar{I}_{\text {down }}$ are the time averaged ion saturation currents measured by two opposed plates of a Mach probe. The factor $K$ is a constant parameter that depends on the ratio between diffusivity and cross-field viscosity. The fluid approach leading to Eq. (1) is valid if the ion sound Larmor radius $\left(T_{i} \simeq 0\right)$ is much smaller than the probe size $\left(\rho_{s} \ll \ell\right)$. Moreover, the ion-neutral mean free path has to be shorter than the collection presheath length $\left(l_{i-n} \ll L_{c}\right) .{ }^{10}$ Both conditions are satisfied for typical TORPEX plasmas since we have $\rho_{s} \sim 3 \times 10^{-4} \mathrm{~m}$ for hydrogen plasmas with $T_{e} \sim 5 \mathrm{eV}, \ell=1 \mathrm{~cm}, l_{i-n} \sim 30 \mathrm{~cm}$, and $L_{c} \sim 10 \mathrm{~m}$. For the factor $K$, we choose a typical value used for Mach probe measurements in the tokamak SOL: $K=0.4 .{ }^{10}$ Moreover, it has be shown that the effect of suprathermal electrons on ion satu- 

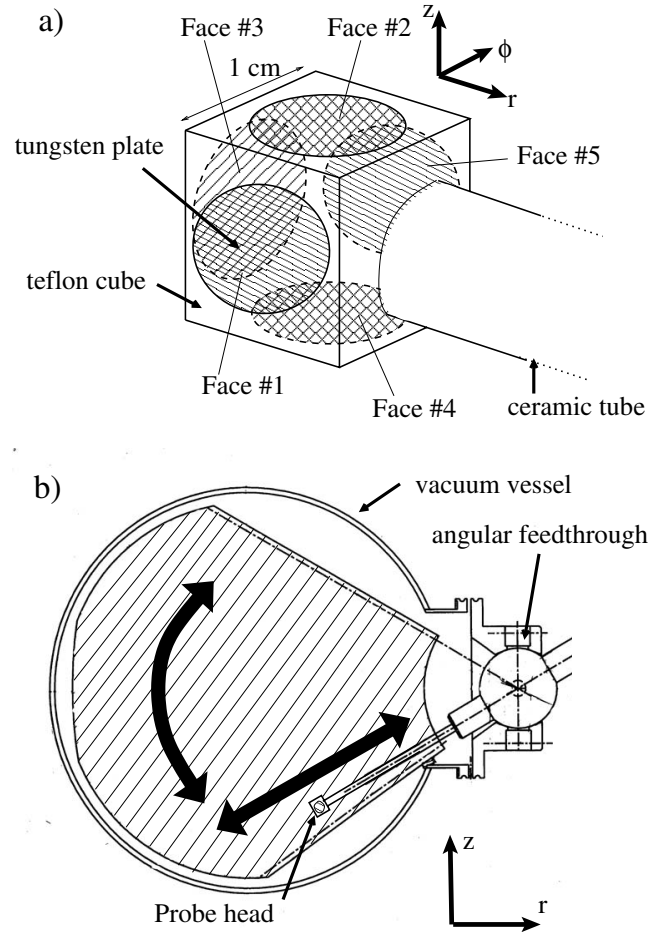

FIG. 1. (a) Drawing of the Mach probe used in these experiments with the face numbering. (b) Schematic of the TORPEX cross section showing the 2D movable system and the covered area.

ration current measurements can be neglected for sufficiently negative bias voltages $\left(V_{\text {bias }} \lesssim-30 \mathrm{~V}\right){ }^{11}$ Therefore, we assume that suprathermal electrons do not affect the Mach number measurements. This is reasonable since it has been predicted $^{12}$ that flow measurements could be affected by suprathermals when their fraction exceeds $10 \%$, which is usually not the case for TORPEX plasmas. ${ }^{13}$

As already mentioned, we would like to know the time evolution of the toroidal Mach number $M_{\phi}$ and of the ion sound speed $C_{s}$ at the same spatial location. This requires to know the time evolution of the ion saturation current and the electron temperature measured on each tip of the Mach probe. The idea is to apply a varying voltage on both sides of the Mach probe and then, using a conditional sampling method, ${ }^{3}$ to reconstruct the $I-V$ Langmuir characteristic over many blob events detected with a reference Langmuir probe, for each tip. A similar method has already been successfully applied to density, temperature, and plasma potential measurements with a standard Langmuir probe. ${ }^{14}$ Therefore, more details of the technique can be found in this reference. Tips 1 and 5 of the Mach probe are swept at $1 \mathrm{kHz}$ frequency in the range $V_{\mathrm{pr}}=[-40,+10] \mathrm{V}$ and the probe current $I_{\mathrm{pr}}$ is measured (tip 3 is biased at $-40 \mathrm{~V}$; the last two are kept floating). A reference probe located at $r=17.5 \mathrm{~cm}$, $z=-2.5 \mathrm{~cm}$, and toroidally displaced by $\Delta \Phi=-30^{\circ}$ is also biased at $V_{\text {ref }}=-40 \mathrm{~V}$. It measures the ion saturation current $I_{\text {ref }}$, characterized by spikes due to the transit of blobs. The threshold is set to $I_{\text {ref }}>3.5 \sigma$, where $\sigma$ is the standard deviation of the reference signal. In the present experiments, $2.5 \mathrm{~s}$ long plasmas are analyzed, providing more than 500 triggers for the chosen threshold. This is enough to ensure a good reconstruction and fit of the $I-V$ characteristic. Finally, by moving the Mach probe on a shot-to-shot basis, one could

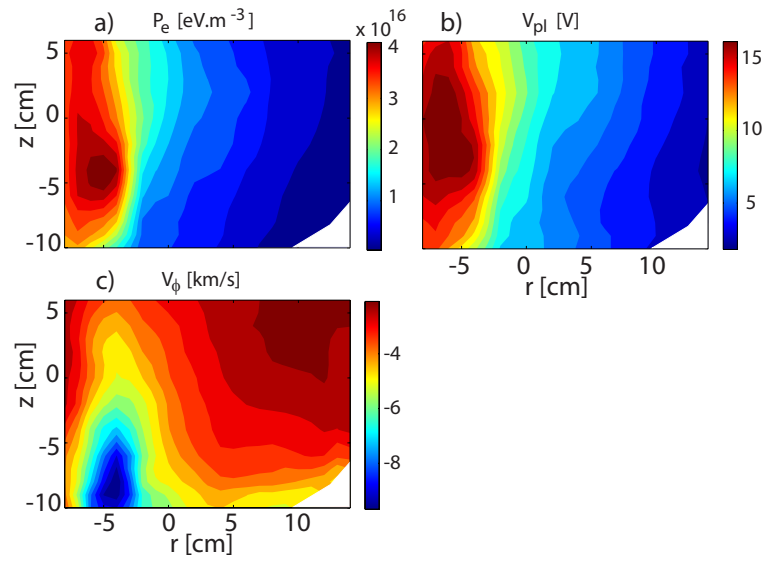

FIG. 2. (Color online) 2D time averaged profiles obtained with the presented method of (a) the electron pressure, (b) the plasma potential, and (c) the toroidal velocity.

obtain time resolved profiles of the toroidal velocity over the poloidal cross section. To the best of the authors knowledge, this is the first time such information is being reported.

The success of the method depends on the quality of the fit of the $I-V$ curve from which we extract the density, the electron temperature, and the plasma potential. As a first step, we look at the time averaged profiles of these plasma parameters. 2D profiles have been reconstructed on a shotto-shot basis scan of the Mach probe position over a $22 \times 16 \mathrm{~cm}^{2}$ rectangle with a spatial resolution of $2 \mathrm{~cm}$. The 2D profiles averaged in time and over the two faces of the plasma pressure, the plasma potential, and the toroidal velocity are shown in Fig. 2. Both plasma pressure and plasma potential profiles are elongated in the vertical direction as it is expected for large vertical magnetic fields. The profiles are in a quantitative agreement with profiles measured with an array of usual Langmuir probes (see Fig. 1 in Ref. 14). The plasma is characterized by coherent fluctuations at $\sim 4 \mathrm{kHz}$ in the pressure gradient region associated with an interchange mode. Moreover, the $\mathbf{E} \times \mathbf{B}$ velocity shear is sufficient to create blobs according to the mechanism proposed in Ref. 15. The 2D time averaged profile of the toroidal velocity is less slablike. Parallel currents, if any, might play a role to explain the observed profile. The complete characterization of the time average profiles of the toroidal velocity is out of the scope of the present paper and will be investigated in dedicated future experiments. Nevertheless, we have found that measured flows in TORPEX are not inconsistent with the background $\mathbf{E} \times \mathbf{B}$ flows. The sign of the toroidal velocity indicates the direction of the flow with respect to the magnetic field: in that case, the negative sign denotes that the plasma flows in the opposite direction compared to $\mathbf{B}$ (i.e., clockwise viewing TORPEX from top).

One important advantage of the CS method is that time resolved measurements can be obtained, keeping in mind that the dynamics is related to the selection of coherent events, plasma blobs in our case. The time evolution of the estimated plasma parameters is reported in Fig. 3. In Figs. 3(a) and 3(b), the electron density $n_{e}$ measured by tips 1 and 5 is compared with the density estimated with the usual conditional average sampling from $I_{\text {sat }}$ measured on tip 3. The interchange mode and the density blob are well captured by the Mach probe. The difference between the time averaged 

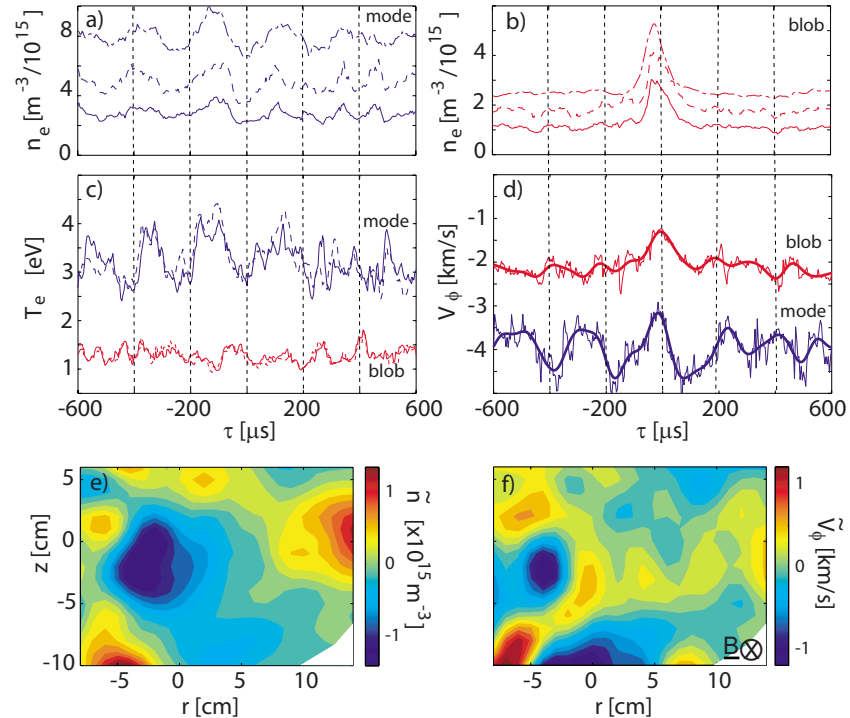

FIG. 3. (Color online) Time evolution of (a) the electron density in the mode region $(r=0 \mathrm{~cm}, z=-4 \mathrm{~cm})$ measured by 1 (solid), 5 (dashed), and 3 (dash dotted), and (b) in the blob region $(r=12 \mathrm{~cm}, z=0 \mathrm{~cm}$ ); (c) the electron temperature measured in both regions by 1 (solid) and 5 (dashed); (d) the toroidal velocity measured in both regions. The thick lines are data filtered with a low-pass filter at $10 \mathrm{kHz}$. Example of a 2D snapshot taken when the blob of (e) the density fluctuations and (f) the toroidal velocity fluctuations is detected $(\tau=0)$.

$n_{e}$ on both sides of the Mach probe already indicates the existence of a parallel flow. The level of fluctuations is of the same order on both faces. The electron temperature is remarkably similar on both sides of the Mach probes [Fig. $3(\mathrm{c})$ ]. The interchange mode is still visible while there is no signature of the blob in $T_{e}$. This is consistent with the fact that the temperature is in phase with the plasma potential and not with the density as shown in Ref. 16.

In Fig. 3(d), the time evolution of the toroidal velocity in the mode region and at the edge is represented. The coherent oscillations associated with the interchange mode are clearly visible and highlighted by the thick line, which corresponds to the low-pass filtered signal. It has to be noted that $V_{\phi}$ and $n$ are evolving almost out of phase. In the edge region, we see that when a density blob is detected, the toroidal velocity decreases a few later. So, a positive density burst $\tilde{n}$ carries a positive $\widetilde{V}_{\phi}$ burst (a positive fluctuation on top of the negative averaged $\bar{V}_{\phi}$ ). The perturbation associated with the density blob on the toroidal velocity is much weaker $(\sim 20 \%)$ than on the density itself $(\sim 80 \%)$. Finally, Figs. 3(e) and 3(f) show the 2D profile of the fluctuating density and toroidal velocity when the blob is detected $(\tau=0)$. The mode is visible around $r=-5 \mathrm{~cm}$ and the blob is seen at the very edge $(r=14 \mathrm{~cm})$.

In summary, we have presented a new method to analyze Mach probe signals. It is based on the conditional sampling technique, the triggering events being plasma density blobs. On a shot-to-shot basis, 2D time resolved profiles of the toroidal plasma velocity are measured in TORPEX plasmas. Following the approach initiated by Hidalgo et al.,${ }^{17}$ detailed investigations of the coupling between the propagation of plasma blobs and the toroidal velocity are under way and will be subject to future publications.

This work is partly funded by the Fonds National Suisse de la Recherche Scientifique.

${ }^{1}$ G. Proudfoot, P. J. Harbour, J. Allen, and A. Lewis, J. Nucl. Mater. 128, 180 (1984).

${ }^{2}$ A. S. Wan, T. F. Yang, B. Lipschultz, and B. Labombard, Rev. Sci. Instrum. 57, 1542 (1986).

${ }^{3}$ H. L. Pecseli and J. Trulsen, Phys. Fluids B 1, 1616 (1989).

${ }^{4}$ A. Fasoli, B. Labit, M. McGrath, S. H. Müller, G. Plyushchev, M. Podestà, and F. M. Poli, Phys. Plasmas 13, 055902 (2006).

${ }^{5}$ M. Podestà, A. Fasoli, B. Labit, M. McGrath, S. H. Müller, and F. M. Poli, Plasma Phys. Controlled Fusion 47, 1989 (2005); 48, 1053 (2006).

${ }^{6}$ G. Plyushchev, A. Diallo, A. Fasoli, I. Furno, B. Labit, S. H. Müller, M. Podestà, F. M. Poli, H. Boemer, W. W. Heidbrink, and Y. Zhang, Rev. Sci. Instrum. 77, 10F503 (2006).

${ }^{7}$ P. Stangeby, The Plasma Boundary of Magnetic Fusion Devices (IOP, Bristol, 2000).

${ }^{8}$ K.-S. Chung, I. H. Hutchinson, B. Labombard, and R. W. Conn, Phys. Fluids B 1, 2229 (1989).

${ }^{9}$ K.-S. Chung, Jpn. J. Appl. Phys., Part 1 45, 10A (2006).

${ }^{10}$ I. H. Hutchinson, Phys. Rev. A 38, 4721 (1988).

${ }^{11}$ M. Podestà, "Plasma production and transport in a simple magnetised toroidal plasma," Ph.D. thesis, EPFL, 2007.

${ }^{12}$ J. P. Gunn and V. Fuchs, Phys. Plasmas 14, 032501 (2007).

${ }^{13}$ M. Podestà, A. Diallo, A. Fasoli, I. Furno, B. Labit, S. H. Müller, and F. M. Poli, Plasma Phys. Controlled Fusion 49, 175182 (2007).

${ }^{14}$ I. Furno, B. Labit, A. Fasoli, F. M. Poli, P. Ricci, C. Theiler, S. Brunner, A. Diallo, J. P. Graves, M. Podestà, and S. H. Müller, Phys. Plasmas 15, 055903 (2008)

${ }^{15}$ I. Furno, B. Labit, M. Podestà, A. Fasoli, S. H. Müller, F. M. Poli, P. Ricci, C. Theiler, S. Brunner, A. Diallo, and J. P. Graves, Phys. Rev. Lett. 100, 055004 (2008).

${ }^{16}$ M. Podestà, A. Fasoli, B. Labit, I. Furno, P. Ricci, F. M. Poli, A. Diallo, S. H. Müller, and C. Theiler, "Cross-field transport by instabilities and blobs in a magnetized toroidal plasma," Phys. Rev. Lett. (to be published).

${ }^{17}$ C. Hidalgo, B. Gonçalves, C. Silva, M. A. Pedrosa, K. Erents, M. Hron, and G. F. Matthews, Phys. Rev. Lett. 91, 065001 (2003). 